UWThPh-2000-27

ESI-979

June 29, 2000

\title{
Two-dimensional anyons and the temperature dependence of commutator anomalies *
}

\author{
Nevena Ilieva ${ }^{*, \sharp}$ \\ Institut für Theoretische Physik \\ Universität Wien \\ and \\ Erwin Schrödinger International Institute \\ for Mathematical Physics
}

\begin{abstract}
The temperature dependence of commutator anomalies is discussed on the explicit example of particular (anyonic) field operators in two dimensions. The correlation functions obtained show that effects of the non-zero temperature might manifest themselves not only globally but also locally.
\end{abstract}

PACS numbers: 03.70.+k, 11.10.Kk, 11.10.Wx, 71.10.Pm

Keywords: fractional statistics, noncanonical fermions, thermal correlators, quantum Hall effect

* Work supported in part by "Fonds zur Förderung der wissenschaftlichen Forschung in Österreich" under grant P11287-PHY;

* On leave from Institute for Nuclear Research and Nuclear Energy, Bulgarian Academy of Sciences, Boul.Tzarigradsko Chaussee 72, 1784 Sofia, Bulgaria

\# E-mail address: ilieva@ap.univie.ac.at 


\section{Introduction}

Anomalies play an important role in quantum field theory. Originally, they have been considered at zero temperature and it is therefore of interest to clarify whether they change upon heating. There is a belief, strengthened also by particular examples (as the usual Schwinger term in the current commutator in two dimensions, [1]), that this is not the case, that is - anomalies remain temperature independent also in a thermal state. A rough argumentation might refer to the fact that anomalies being a typically local phenomenon, should not be affected by global features of the theory, such as the temperature.

That this is not necessarily so will be demonstrated on the explicit example of particular field operators in two dimensions whose correlation functions exhibit severe temperature dependence, so that finite-temperature effects manifest themselves not only globally but also locally.

\section{The anyonic field operators}

In the discussion of Bose-Fermi duality at finite temperature there appear in a natural way field operators with exotic exchange relations, so anyons [2, 3]

$$
\Psi_{\alpha}(x):=\lim _{\varepsilon \rightarrow 0} n_{\alpha}(\varepsilon) \exp \left[i \sqrt{2 \pi \alpha} \int_{-\infty}^{\infty} d y \varphi_{\varepsilon}(x-y) j(y)\right]
$$

with $n_{\alpha}$ - some renormalization parameter and $\varphi_{\varepsilon}(x)$ - an approximation to the Heaviside function

$$
\lim _{\varepsilon \rightarrow 0} \varphi_{\varepsilon}(x)=\Theta(x), \quad \varphi(x) \in H_{1},
$$

where $H_{1}$ is the Sobolev space, $H_{1}=\left\{f: f, f^{\prime} \in L^{2}\right\}$. Two special families of such operators are distinguished: those characterized by statistic parameter $\alpha=2 \cdot 2 n \pi$ being actually bosons and those characterized by statistic parameter $\alpha=2(2 n+1) \pi$ being respectively fermions (in both cases $n \in \mathbf{Z}$ ). All operators (1.1) are goverened by one and the same dynamical equation — Heisenberg's Urgleichung (pre-equation) [四]

$$
\not \partial \psi(x)=\lambda \psi(x) \bar{\psi}(x) \psi(x), \quad \lambda=\sqrt{2 \pi \alpha} .
$$

With no bosons present in it at all, Eq.(1.2) represents the ultimate version of the opinion that fermions should enter the basic formalism of a fundamental theory of elementary particles, that is usually taken for granted.

Thus, the coupling constant in Heisenberg's pre-equation turns out to be related to the statistic parameter, that characterizes different anyons. Since the latter are uncountably many and live in orthogonal spaces, the whole Hilbert space becomes nonseparable and in each of its sectors a different pre-equation holds - a feature that certainly cannot be seen by any power expansion in $\lambda$, which, however, in the above context becomes problematic itself. 
Investigation of anyonic field operators of the type (1.1) represents by far not only an academic interest - such fields might become of importance in solid-state physics, in problems like quantum wires and FQHE. The relation between the objects there involved and the field operators (1.1) is rather obvious.

Recall [2] that being governed by Heisenberg's pre-equation means that anyons (1.1) are all solutions to the (finite-temperature) Thirring model [5]. On the other hand, it is well known [6, 7] that a one-dimensional (interacting) electron gas is effectively described by the Luttinger model - a Thirring-like model, in which the current-current interaction is not strictly localized but is characterized by a suitable form-factor [8]

$$
H_{L u t}=i \psi_{L}^{\dagger} \partial_{x} \psi_{L}+i \psi_{R}^{\dagger} \partial_{x} \psi_{R}+\int \psi_{L}^{\dagger} \psi_{L}(x) V(x-y) \psi_{R}^{\dagger} \psi_{R}(y) d y .
$$

Actually, for repulsive interactions Hamiltonian (1.3) is driven by renormalization group flow to the Thirring-model one, $V(x-y) \rightarrow g \delta(x-y)$, with an effective coupling constant $g$, so that the corresponding bosonised Hamiltonian takes the form

$$
H_{L u t}=\frac{h v_{F}}{4} \int d x:\left(\frac{1}{v_{F}^{2}}\left(\partial_{t} \Phi\right)^{2}+(1+g)\left(\partial_{x} \Phi\right)^{2}\right):,
$$

where : : stands for Wick's ordering, $v_{F}$ is the Fermi velocity, $n=\partial_{x} \Phi$ is the particle dencity and $I=e \partial_{t} \Phi$ - the electric current.

The Hamiltonian (1.4) describes two different physical systems, a quantum wire and the edges of a Quantum Hall bar. These systems differ by the form of creation and annihilation operators of physical electrons. Since the interaction with the external reservoirs is via electron exchange, the precise form of the electron operators is crucial for computing the longitudinal conductance in quantum wires and Hall conductance in the Quantum Hall setting [9]. In the case of quantum wires, one obtains a one-parameter family of CFT's, with non-chiral electron operators and non-renormalized (independent on $g$ ) conductance value, $\sigma=I / V=e^{2} / h$. In the case of QH fluids, the electrons are chiral because the two edges are separated by a macroscopically big sample. The Quantum Hall filling fraction is related to the effective Luttinger coupling by formula,

$$
\nu=\frac{1}{\sqrt{1+g}}
$$

Hence, the Hall conductance is reduced with respect to the fundamental unit of $e^{2} / h$, $\sigma_{H}=\nu e^{2} / h$. In fact, the incompressibility of the Quantum Hall fluid requires $\nu^{-1}$ be an odd integer, such that $\sqrt{1+g}=2 k+1, k \in \mathbf{Z}$. The case of $k=0$ corresponds to the integer Quantum Hall effect, $\nu=1$, and the noninteracting Luttinger model with $g=0$ is recovered. In the case of $k \neq 0$ one obtains Laughlin's filling fraction [10] in the theory of FQHE. In this case, the physical electrons are identified with Wen's fermions [11, 12] and have an unusual statistics which depends on the filling fraction.

These edge-excitation operators are special cases of anyons (1.1). However, in the fermionic case - $\alpha=2 \pi(2 n+1)$, so for Laughlin's states - one has to distinguish between fermions, corresponding to $n=0$ and $n \neq 0$. These fields, though locally anticommuting, are severely different: the former are canonical fields, while the latter are not and this difference shows up also in their thermal properties as will be argued below. 


\section{Commutator anomalies and algebra extensions}

The first known commutator anomaly — the one in the current commutator in two dimensions, has been discovered in the thirties by Jordan [13] and Born [14], in the attempts for construction of neutrino theory of light. Then the observation has been made that because of the unboundedness from below of the free-fermion Hamiltonian fermionic creation and annihilation operators should undergo what is called now a Bogoliubov transformation. Thus the stability of the system is achieved but in addition an anomalous term (later called "Schwinger term") appeares in the current commutator, so that for the smeared currents one gets

$$
\left[j_{f}, j_{g}\right]=\int_{-\infty}^{\infty} \frac{d p}{(2 \pi)^{2}} p \tilde{f}(p) \widetilde{g}(-p)=\frac{i}{2 \pi} \int_{-\infty}^{\infty} d x f^{\prime}(x) g(x)=i \sigma(f, g)
$$

$\sigma(f, g)$ being the symplectic form on the current algebra $\mathcal{A}_{c}$. The question is how far is this result state- (so, representation-) dependent.

The rigorous definition of the anyonic field operators proposed by us [2, 3] allows for a detailed analysis of such questions. It is based on the construction of a chain of algebraic inclusions, starting with the CAR-algebra of bare fermions $\mathcal{A}$,

$$
\operatorname{CAR}(\text { bare }) \subset \pi_{\beta}(\mathcal{A})^{\prime \prime} \supset \mathcal{A}_{c} \subset \overline{\mathcal{A}}_{c} \subset \bar{\pi}_{\beta}\left(\overline{\mathcal{A}}_{c}\right)^{\prime \prime} \supset \operatorname{CAR}(\text { dressed }) .
$$

The shift $\tau_{t}$ is an automorphism of $\mathcal{A}$ which has KMS-states $\omega_{\beta}$ and associated representations $\pi_{\beta}$. In $\pi_{\beta}(\mathcal{A})^{\prime \prime}$ one finds bosonic modes $\mathcal{A}_{c}$ with an algebraic structure independent on $\beta$. The crucial ingredient needed so far was the appropriately chosen state. We make use of the KMS-state (which is unique for the shift over the CAR algebra). Another possibility would be to introduce the Dirac vacuum (filling all negative energy levels in the Dirac sea). This is what has been originally done in the thirties [13, 14], and recovered later by Mattis and Lieb [6] in the context of the Luttinger model. However, an important detail might be overseen that way: symplectic structure (2.1) though formally independent on $\beta$ (see also [1]), for $\beta<0$ changes its sign, $\sigma \rightarrow-\sigma$, and for $\beta=0$ (the tracial state) becomes zero, that is

$$
\left[j_{f}, j_{g}\right]=i \sigma(f, g)=\left\{\begin{array}{cc}
\int f^{\prime} g & \text { for } \beta>0 \\
0 & \text { for } \beta=0 \\
-\int f^{\prime} g & \text { for } \beta<0
\end{array}\right.
$$

Remark. It is the parity $P$ (which suffers a destruction on the passage from the CARalgebra to the current algebra [15, 3]) that relates the states corresponding to positive and negative temperatures

$$
\omega_{-\beta}=\omega_{\beta} \circ P
$$

In the construction of algebraic chain (2.2) we make use of the $\tau$-KMS states, which are translation-invariant equilibrium states at an inverse temperature $\beta$. On $\mathcal{A}_{c}$ such a state is given by the two-point function

$$
\omega(j(f) j(g))=\int d x d y w(x-y) f(x) g(y),
$$


with a kernel

$$
w(x-y)=-\lim _{\varepsilon \rightarrow 0^{+}} \frac{1}{(2 \pi)^{2} \operatorname{sh}^{2}(\mathrm{x}-\mathrm{y}-\mathrm{i} \varepsilon)} .
$$

Recall that for Weyl operators, the multiplication law replaces relation (2.1)

$$
e^{i j(f)} e^{i j(g)}=e^{\frac{i}{2} \sigma(g, f)} e^{i j(f+g)} .
$$

with $\sigma$ - the symplectic form on the algebra. The expectation of the Weyl operators is given by

$$
\omega\left(e^{i j(f)}\right)=e^{-\frac{1}{2}\langle f \mid f\rangle},
$$

where the scalar product $\langle f \mid g\rangle$ defines the one-particle real Hilbert space $h$ of the $f$ 's. For consistency, it has to satisfy

$$
\sigma(f \mid g)=(\langle g \mid f\rangle-\langle f \mid g\rangle) / 2 \text {. }
$$

Eqs.(2.5),(2.6) imply

$$
\omega\left(e^{i j(f)} e^{i j(g)}\right)=\exp \left\{-\frac{1}{2}[\langle f \mid f\rangle+\langle g \mid g\rangle+2\langle f \mid g\rangle]\right\},
$$

or generally

$$
\omega\left(\prod_{k} e^{i j\left(f_{k}\right)}\right)=\exp \left\{-\frac{1}{2}\left[\sum_{k}\left\langle f_{k} \mid f_{k}\right\rangle+2 \sum_{k<m}\left\langle f_{k} \mid f_{m}\right\rangle\right]\right\} .
$$

\section{The KMS correlation functions}

The anyons (1.1) are Weyl operators for which the smearing function is $f_{\alpha}^{x}(y)=$ $\sqrt{2 \pi \alpha} \Theta(x-y)$. So far $\mathcal{A}_{c}$ was defined for $j_{f}$ 's with $f \in C_{0}^{\infty}$, for instance, that is with functions which vanish for $x \rightarrow \pm \infty$. The structure of $\mathcal{A}_{c}$ is determined by the symplectic form $\sigma(f, g)$ (2.1) which is actually well defined also for the Sobolev space, $\sigma(f, g) \rightarrow \sigma(\bar{f}, \bar{g}), \bar{f}, \bar{g} \in H_{1}, H_{1}=\left\{f: f, f^{\prime} \in L^{2}\right\}$. Also $\bar{\omega}_{\beta}$ can be extended to $H_{1}$, since $\bar{\omega}_{\beta}\left(e^{i j_{\bar{f}}}\right)>0$ for $\bar{f} \in H_{1}$. Thus, the symplectic form (2.1) may be given a sense for functions that tend to a constant, however they cannot be reached as limits of functions from $\mathcal{C}_{0}^{\infty}$. For instance,

$$
\begin{gathered}
\Phi_{\delta, \varepsilon}(x):=\varphi_{\varepsilon}(x)-\varphi_{\varepsilon}(x+\delta) \in H_{1}, \\
\lim _{\substack{\delta \rightarrow \infty \\
\varepsilon \rightarrow 0}} \Phi_{\delta, \varepsilon}(x)=\Theta(x)
\end{gathered}
$$

with

$$
\varphi_{\varepsilon}(x):=\left\{\begin{array}{cl}
1 & \text { for } x \leq-\varepsilon \\
-x / \varepsilon & \text { for }-\varepsilon \leq x \leq 0 \\
0 & \text { for } x \geq 0
\end{array}\right.
$$


does not work, since $\sigma\left(\Phi_{x, \delta}, \Phi_{x^{\prime}, \delta^{\prime}}\right)$ depends on the order in which the limits $\delta, \delta^{\prime} \rightarrow \infty$ are taken and only for $\delta=\delta^{\prime} \rightarrow \infty$ we get the desired result $i \operatorname{sign}\left(\mathrm{x}-\mathrm{x}^{\prime}\right)$. Since this appears in the $c$-number part, in no representation can $j\left(\Phi_{x, \delta}\right)$ converge strongly. Nevertheless, for functions with the same (nontrivial) asymptotics at, say, $x \rightarrow \infty$ and whose difference $\in h$ one can succeed in getting the expectation values as limits. In the case when different anyonic contributions "compensate" each other a nontrivial result is obtained due to the cancellation of the infrared diveregencies. In terms of $\Psi$ 's this means that the expectation value of a product of $\Psi$ 's and $\Psi^{*}$ 's is different from zero only if there are as many $\Psi$ 's as $\Psi^{*}$ s, equivalently - if the "total" statistic parameter of creation operators equals the one of annihilation operators, for instance $\left\langle\Psi_{1}^{*} \Psi_{1}^{*} \Psi_{4}\right\rangle \neq 0$, or otherwise that they lead to orthogonal sectors of the enlarged Hilbert space.

Next we have to cope with the ultraviolet problem. Here the situation is different since we do not need a larger Hilbert space to this end. The solution is already contained in the usage of a smeared step function in (3.1). A trivial integration then gives, e.g. for the $\alpha$ two-point function $\left(F_{x, \varepsilon}^{\alpha}:=\sqrt{\alpha} \varphi_{\varepsilon}(x)\right)$

$$
\begin{array}{r}
\omega\left(\Psi_{\alpha}^{*}(x) \Psi_{\alpha}(y)\right)=\omega\left(e^{-i j\left(F_{x, \varepsilon}^{\alpha}\right)} e^{i j\left(F_{y, \varepsilon}^{\alpha}\right)}\right. \\
=\left(\frac{i \varepsilon}{\operatorname{sh} \frac{\pi\left(\mathrm{x}-\mathrm{x}^{\prime}-\mathrm{i} \varepsilon\right)}{\beta}}\right)^{(\alpha / 2 \pi)} .
\end{array}
$$

The divergence for $\varepsilon \rightarrow 0$ remains and determines the neccessary renormalization of the operators $\Psi_{\alpha}$ [3]. Thus, for $\alpha=2 \pi$ the renormalization parameter should be $n_{2 \pi}(\varepsilon)=(2 \pi \varepsilon)^{-1 / 2}$, so that the fermion KMS two-point function is recovered, and for the general case of an arbitrary $\alpha: n_{\alpha}(\varepsilon)=(2 \pi \varepsilon)^{-\alpha / 4 \pi}=n_{2 \pi}^{\alpha}(\varepsilon)$.

For all $\alpha$ 's the two-point function (for $x>x^{\prime}$ and $\beta=\pi$ )

$$
\left\langle\Psi_{\alpha}^{*}(x) \Psi_{\alpha}\left(x^{\prime}\right)\right\rangle_{\beta}=\left\langle\Psi_{\alpha}(x) \Psi_{\alpha}^{*}\left(x^{\prime}\right)\right\rangle_{\beta}=\left(\frac{i}{2 \beta \sinh \left(x-x^{\prime}\right)}\right)^{\alpha / 2 \pi}=: S_{\alpha}\left(x-x^{\prime}\right)
$$

has the desired properties

1. Hermiticity:

$$
S_{\alpha}^{*}(x)=S_{\alpha}(-x) \Longleftrightarrow\left\langle\Psi_{\alpha}^{*}(x) \Psi_{\alpha}\left(x^{\prime}\right)\right\rangle_{\beta}^{*}=\left\langle\Psi_{\alpha}^{*}\left(x^{\prime}\right) \Psi_{\alpha}(x)\right\rangle_{\beta}
$$

2. $\alpha$-commutativity:

$$
S_{\alpha}(-x)=e^{i \alpha / 2} S_{\alpha}(x) \Longleftrightarrow\left\langle\Psi_{\alpha}\left(x^{\prime}\right) \Psi_{\alpha}^{*}(x)\right\rangle_{\beta}=e^{i \alpha / 2}\left\langle\Psi_{\alpha}^{*}(x) \Psi_{\alpha}\left(x^{\prime}\right)\right\rangle_{\beta} ;
$$

3. KMS-property:

$$
S_{\alpha}(x)=S_{\alpha}(-x+i \pi) \Longleftrightarrow\left\langle\Psi_{\alpha}^{*}(x) \Psi_{\alpha}\left(x^{\prime}\right)\right\rangle_{\beta}=\left\langle\Psi_{\alpha}\left(x^{\prime}\right) \Psi_{\alpha}^{*}(x+i \pi)\right\rangle_{\beta} .
$$


For $\alpha=2 \pi$ Eq.(5.7) reads

$$
\left\langle\Psi_{2 \pi}^{*}(x) \Psi_{2 \pi}\left(x^{\prime}\right)\right\rangle_{\beta}=\lim _{\varepsilon \rightarrow 0_{+}} \frac{i}{2 \beta \sinh \frac{\pi\left(x-x^{\prime}-i \varepsilon\right)}{\beta}} .
$$

For $f(y) \rightarrow \Theta(x-y), g\left(y^{\prime}\right) \rightarrow-\Theta\left(x^{\prime}-y^{\prime}\right)$ nothing changes so we verify the relation $(5.2) \leftrightarrow(5.3)$

$$
\left\langle\Psi_{2 \pi}^{*}(x) \Psi_{2 \pi}\left(x^{\prime}\right)\right\rangle_{\beta}=\left\langle\Psi_{2 \pi}(x) \Psi_{2 \pi}^{*}\left(x^{\prime}\right)\right\rangle_{\beta} .
$$

For $\alpha=4 \pi$ we get like for the $j$ 's

$$
\left\langle\Psi_{4 \pi}^{*}(x) \Psi_{4 \pi}\left(x^{\prime}\right)\right\rangle_{\beta}=-\frac{1}{\left(2 \beta \sinh \frac{\pi\left(x-x^{\prime}-i \varepsilon\right)}{\beta}\right)^{2}},
$$

whereas for $\alpha=6 \pi$ we get a different kind of fermions

$$
\left\langle\Psi_{6 \pi}^{*}(x) \Psi_{6 \pi}\left(x^{\prime}\right)\right\rangle_{\beta}=-\frac{i}{\left(2 \beta \sinh \frac{\pi\left(x-x^{\prime}-i \varepsilon\right)}{\beta}\right)^{3}} .
$$

This shows that local anticommutativity alone does not guarantee the uniqueness of the KMS-state, one needs in addition the CAR-relations. The $\Psi_{\alpha}$ 's, $\alpha \in 2(2 \mathbf{N}+1) \pi$, describe an infinity of inequivalent fermions.

Thus we find a kind of a quantum selection rule for our original dynamical equation - Heisenberg's pre-equation. It admits fermionic solutions only for $\lambda=\sqrt{2 \pi}$. Of course one could for any $\lambda$ enforce Fermi statistics by renormalizing the bare fermion field $\psi \rightarrow \sqrt{Z} \psi, j \rightarrow Z j$ with a suitable $Z(\lambda)$ but this just means pushing factors around. Also for the series of values $\lambda=\sqrt{2(2 n+1) \pi}, n \in \mathbf{Z}^{+}, n \neq 0$ one gets anticommuting fields as solutions, however they are noncanonical.

These non-canonical Fermi-fields are similar to Wen's fermions

$$
\left\langle\psi(z) \psi^{\dagger}(w)\right\rangle \sim \frac{1}{(z-w)^{2 n+1}}
$$

which correspond to Laughlin's plateaux in the theory of the FQHE, but considered at finite temperature. For a detailed analysis of this relation we refer to [16].

In complete analogy, for the $n$-point function we obtain

$$
\begin{aligned}
& \omega\left(\Psi_{\alpha}^{*}\left(x_{1}\right) \ldots \Psi_{\alpha}^{*}\left(x_{n}\right) \Psi_{\alpha}\left(y_{n}\right) \ldots \Psi_{\alpha}\left(y_{1}\right)\right) \\
= & \frac{\prod_{k>l}\left(\operatorname{sh}\left(\mathrm{x}_{\mathrm{k}}-\mathrm{x}_{\mathrm{l}}-\mathrm{i} \varepsilon\right)\right)^{\alpha / 2 \pi} \prod_{\mathrm{k}>\mathrm{l}}\left(\operatorname{sh}\left(\mathrm{y}_{\mathrm{k}}-\mathrm{y}_{\mathrm{l}}-\mathrm{i} \varepsilon\right)\right)^{\alpha / 2 \pi}}{\prod_{k, l}\left(-2 \pi i \operatorname{sh}\left(\mathrm{x}_{\mathrm{k}}-\mathrm{y}_{\mathrm{l}}-\mathrm{i} \varepsilon\right)\right)^{\alpha / 2 \pi}} .
\end{aligned}
$$

The exact exchange relations are hidden in the factor $i^{\alpha}$ in (3.7) and we shall return to their detailed analysis later on. 
For the case $\alpha=2 \pi$ with the help of Cauchy's determinant formula it can be rewritten as

$$
\frac{\prod_{i>k} \operatorname{sh}\left(\mathrm{x}_{\mathrm{i}}-\mathrm{x}_{\mathrm{k}}-\mathrm{i} \varepsilon\right) \prod_{\mathrm{i}>\mathrm{k}} \operatorname{sh}\left(\mathrm{y}_{\mathrm{i}}-\mathrm{y}_{\mathrm{k}}-\mathrm{i} \varepsilon\right)}{\prod_{i, k} \operatorname{sh}\left(\mathrm{x}_{\mathrm{i}}-\mathrm{y}_{\mathrm{k}}-\mathrm{i} \varepsilon\right)}=\operatorname{Det} \frac{1}{\operatorname{sh}\left(\mathrm{x}_{\mathrm{i}}-\mathrm{y}_{\mathrm{k}}-\mathrm{i} \varepsilon\right)},
$$

so we recover the original free fermions with all their characteristic properties.

\section{Temperature dependence of the $\alpha$-commutator}

For the thermal expectation of the $\alpha$-commutator one finds

$$
\begin{aligned}
\omega_{\beta}\left(\left[\Psi^{*}(x), \Psi\left(x^{\prime}\right)\right]_{\alpha}\right) & =-i\left(-\frac{1}{2 \beta \sinh \frac{\pi\left(x-x^{\prime}-i \varepsilon\right)}{\beta}}\right)^{\alpha / 2 \pi} \\
& +i\left(-\frac{1}{2 \beta \sinh \frac{\pi\left(x-x^{\prime}+i \varepsilon\right)}{\beta}}\right)^{\alpha / 2 \pi}
\end{aligned}
$$

and for $\varepsilon \rightarrow 0$ a distribution is obtained where only the leading singularity is temperature independant, namely

$$
\lim _{\varepsilon \rightarrow 0}\left\{\left(\frac{1}{x-x^{\prime}-i \varepsilon}\right)^{\alpha / 2 \pi}-\left(\frac{1}{x-x^{\prime}+i \varepsilon}\right)^{\alpha / 2 \pi}\right\}=?
$$

For example, for $\alpha=6 \pi$ this expectation is

$$
\left\langle-\left[\Psi_{6 \pi}^{*}(x), \Psi_{6 \pi}\left(x^{\prime}\right)\right]_{\alpha}\right\rangle_{\beta}=\left\langle\left[\Psi_{6 \pi}^{*}(x), \Psi_{6 \pi}\left(x^{\prime}\right)\right]_{+}\right\rangle_{\beta}=-\frac{1}{8 \pi^{2}}\left(\delta^{\prime \prime}\left(x-x^{\prime}\right)-\frac{\pi^{2}}{\beta^{2}} \delta\left(x-x^{\prime}\right)\right) .
$$

The commutation relations themselves exhibit an operator structure 17]. Thus, while for $\alpha=2 \pi$ the canonical commutation relations are recovered, for $\alpha=4 \pi$ the commutator reads

$$
\left[\Psi_{4 \pi}^{*}(x), \Psi_{4 \pi}(y)\right]=\frac{i}{2 \pi} \delta^{\prime}(x-y)+i \sqrt{2} \delta(x-y) j(x),
$$

which is essentially the Frenkel-Kac representation of the $s u(2)$ current algebra, and for $\alpha=6 \pi$ the anticommutator takes the form

$$
\begin{aligned}
{\left[\Psi_{6 \pi}^{*}(x), \Psi_{6 \pi}(y)\right]_{+} } & =-\frac{1}{8 \pi^{2}}\left[\delta^{\prime \prime}(x-y)-2 \pi \sqrt{3} \delta^{\prime}(x-y) j(x)+12 \pi^{2} \delta(x-y) j^{2}(x)\right] \\
& +\frac{1}{8 \beta^{2}} \delta(x-y)
\end{aligned}
$$

This demonstrates once again the non-canonical nature already of the first extension classes - bosonic, as well as fermionic - beyond the initial Fermi-algebra. The temperature dependece appears with the first non-canonical Fermi-class. Since the algebra 
of the $6 \pi$-fermions $\mathcal{A}_{(3)}:=\left\{\Psi_{\alpha}: \alpha=6 \pi\right\}$ is a subalgebra of $\mathcal{A}_{(1)}:=\left\{\Psi_{\alpha}: \alpha=2 \pi\right\}$, the algebra of the original fermions, this shows that representations that correspond to different temperatures are inequivalent not only globally, but also locally. In turn, this means that the temperature effects in this construction become locally observable.

\section{Conclusions and outlook}

We have discussed the temperature dependence of correlation functions and exchange relations of the two-dimensional anyons constructed in [2, 3]. For the (infinity of inequivalent) anticommuting fields that are present among them, this dependence means a loss of the local normality of representations corresponding to different temperatures, hence (already local) observability of the temperature effects. Consideration of these noncanonical Fermi-fields in detail is of particular interest since their relation to Wen's fermions allows for a rigorous analysis of the possibility for a second-quantization picture of the fractional quantum Hall effect [18].

\section{Acknowledgements}

I appreciate the suggestive discussions with A. Alekseev and L. Georgiev and the useful remarks of K.-H. Rehren on the matter of this note and the fruitful collaboration with H. Narnhofer and W. Thirring on various related topics.

The hospitality and financial support of the International Erwin Schrödinger Institute for Mathematical Physics where part of the research has been performed, are gratefully acknowledged. This work has been supported in part also by "Fonds zur Förderung der wissenschaftlichen Forschung in Österreich" under grant P11287-PHY.

\section{References}

[1] H. Grosse, W. Maderner, C. Reitberger, in Generalized Symmetries in Physics, Eds. H.-D. Döbner, V. Dobrev, and A. Ushveridze (World Scientific, Singapore, 1994).

[2] N. Ilieva, W. Thirring, Eur. Phys. J. C 6, 705 (1999).

[3] N. Ilieva, W. Thirring, Theor. Math. Phys. 121, 1294 (1999).

[4] W. Heisenberg, Z. Naturforsch. 9a, 292 (1954).

[5] W. Thirring, Ann. Phys. 3, 91 (1958).

[6] D.C. Mattis and E. Lieb, J. Math. Phys. 6, 304 (1965).

[7] F.D.M. Haldane, J. Phys. C 14, 2585 (1981). 
[8] J.M. Luttinger, J. Math. Phys 4, 1154 (1963).

[9] A. Alekseev, V. Cheianov, J. Fröhlich, Phys. Rev. B 54, R17320 (1996).

[10] R.B. Laughlin, Phys. Rev. Lett. 50, 1395 (1983); Phys. Rev. B 27, 3383 (1983).

[11] X.G. Wen, Phys. Rev. B41, 12838 (1990).

[12] J. Fröhlich, T. Kerler, Nucl. Phys. B354, 369 (1991).

[13] P. Jordan, Z. Phys. 93, 464 (1935); 98, 759 (1936);99, 109 (1936).

[14] M. Born, N. Nagendra-Nath, Proc. Ind. Acad. Sci. 3, 318 (1936).

[15] N. Ilieva, W. Thirring, in: Problems of QFT, Eds. B.M. Barbashov, G.V. Efimov, A.V. Efremov (E2-99-35, Dubna, 1999) , p.167.

[16] N. Ilieva and W. Thirring, Phys. Lett. B (at press, hep-th/0010030).

[17] N. Ilieva, H. Narnhofer, W. Thirring, "Thermal correlators of anyons in two dimensions", Vienna preprint UWThPh-2000-14, ESI-864 (2000), math-ph/0004006.

[18] N. Ilieva and W. Thirring, "Second quantization picture of the edge currents in the fractional quantum Hall effect", Vienna Preprint UWThPh-2000-37, ESI-953 (2000), math-ph/0010038. 